\title{
New dynamic viewing of mast cells in pulmonary arterial hypertension (PAH): contributors or outsiders to cardiovascular remodeling
}

\author{
Jian Xu ${ }^{1 \#}$, Jingjing Wang ${ }^{2 \#}$, Chengjie Shao ${ }^{1 \#}$, Xiaoning Zeng ${ }^{1}$, Lixiang Sun ${ }^{1}$, Hui Kong ${ }^{1}$, Weiping Xie ${ }^{1}$, \\ Hong Wang ${ }^{1}$
}

${ }^{1}$ Department of Respiratory \& Critical Care Medicine, the First Affiliated Hospital of Nanjing Medical University, Nanjing 210029, China;

${ }^{2}$ Department of Respiratory and Critical Care Medicine, Shanghai Pulmonary Hospital, Tongji University School of Medicine, Shanghai 200433, China

Contributions: (I) Conception and design: J Xu, J Wang, C Shao; (II) Administrative support: W Xie, H Wang; (III) Provision of study materials or patients: X Zeng, H Kong; (IV) Collection and assembly of data: L Sun; (V) Data analysis and interpretation: J Xu, J Wang; (VI) Manuscript writing: All authors; (VII) Final approval of manuscript: All authors.

\#These authors contributed equally to this work.

Correspondence to: Weiping Xie, MD, PhD; Hong Wang, MD, PhD. Department of Respiratory \& Critical Care Medicine, The First Affiliated Hospital of Nanjing Medical University, 300 Guangzhou Road, Nanjing 210029, China. Email: wpxie@njmu.edu.cn; hongwang@njmu.edu.cn.

Background: In patients with pulmonary arterial hypertension (PAH), mast cells (MCs) are extensively observed around pulmonary vessels. However, their temporal and spatial variation during PAH development remains obscure. This study investigated the dynamic evolution of MCs in lungs and right ventricles (RV) to illuminate their role in pulmonary vascular and RV remodeling.

Methods: The PAH model was established by a single intra-peritoneal injection of monocrotaline (MCT, $60 \mathrm{mg} / \mathrm{kg}$ ) in rats. On day 0, 3, 7, 14, and 28 after MCT injection, lung and RV tissues were harvested for staining with hematoxylin and eosin (HE), Gomori aldehyde fuchsin (GAF), toluidine blue (TB) and picrosirius red (PSR). Immunohistochemistry was performed to evaluate the levels of $\alpha$-SMA, CD68 and tryptase. A simple RV remolding model was produced as well by pulmonary artery banding (PAB). RV tissues were collected to determine the degree of MCs infiltration.

Results: After MCT challenge, elevated mean pulmonary arterial pressure (mPAP), increased RV systolic pressure (RVSP), pulmonary arterial media hypertrophy as well as distal vascular muscularization gradually occurred with time. MCs recruitment along with CD68+ macrophages accumulation was observed around distal pulmonary vessels and in alveolar septa. Excessive infiltration and degranulation of MCs were detected in MCT-treated group in lung tissues but not in RV. In addition, no exacerbation of MCs infiltration and degranulation in RV was noted in PAB-treated rats, suggesting few contributions of MCs to RV remodeling. Conclusions: Our findings implied a crucial role of MCs in the remodeling of pulmonary vessels, not $\mathrm{RV}$, which probably through releasing cytokines such as tryptase. The present study enriches the knowledge about PAH, providing a potential profile of MCs as a switch for the treatment of PAH.

Keywords: Mast cells (MCs); pulmonary vascular remodeling; right ventricular remodeling (RV remodeling)

Submitted Jan 27, 2018. Accepted for publication Apr 25, 2018.

doi: $10.21037 /$ jtd.2018.05.59

View this article at: http://dx.doi.org/10.21037/jtd.2018.05.59 


\section{Introduction}

Pulmonary arterial hypertension (PAH) is characterized by a progressive vascular and right ventricular (RV) remodeling, which brings deaths to patients with severe heart failure $(1,2)$. The histopathologic features of pulmonary vascular remodeling include intima injury and repair, medial layer hypertrophy, and adventitia inflammation $(3,4)$. Inflammation and remodeling are considered to connected with each other closely, and this interaction determines the outcome of PAH. Accumulation of mast cells (MCs) in peri-vasculature is crucial to $\mathrm{PAH}$ development. It was first revealed that degranulation of MCs around pulmonary vascular could increase vascular resistance when hypoxia occurred, while no change was observed in MCs density and distribution (5). For Tucker, hypoxia did not change the number of MCs in peribronchial and alveolar septa (6). However, an increase of MCs in alveolar septa and a decrease around bronchia were observable (7). There are also investigations about the redistribution of MCs in lung tissues in the case of acute or chronic hypoxia (8). The level of perivascular chymase+ MCs was higher in patients with pulmonary hypertension (PH) than in donors (9). All these findings highlight the contribution of MCs present in the development of PAH, but no consensus is available about their characteristics till now.

Currently, MCs are attracting increasing attention for their pivotal role in PAH. Given their distribution and degranulation in lungs (e.g., histamine, serotonin, matrix metalloproteinases, tryptase and chymase), MCs are viewed as a candidate for PAH management (10). It was indicated that inhibiting MCs degranulation with disodium cromoglycate attenuated pulmonary vascular remodeling in PAH rats (11). MCs stabilizer cromolyn prevented monocrotaline (MCT)treated rats from vascular injury and repair in lungs $(12,13)$. Inhibition of proteinase signaling such as chymase activity (14) or tryptase/protease-activated receptor 2 (PAR2) pathway (15) could significantly alleviate pulmonary vascular remodeling in PAH. Accumulating data have confirmed the crucial profile of MCs as a potent target for PAH treatment.

However, little is known about the dynamic evolution of MCs in lungs during PAH progression. Since no evidence has so far been provided to assure the function of MCs in $\mathrm{RV}$ remodeling during $\mathrm{PAH}$, we comprehensively investigated the evolution of MCs in lung tissues during PAH development. This study clarified the contribution of MCs in pulmonary vascular and RV remodeling through comparing the density, distribution and degranulation of
MCs at different stages of PAH in lungs and RVs.

\section{Methods}

\section{Etbics statement}

Male Sprague-Dawley (SD) rats $(200 \pm 10 \mathrm{~g})$, purchased from Shanghai Bikai Laboratory Animal Company (Shanghai, China), were raised under standard laboratory conditions with free access to water and food. All procedures were performed according to the National Institutes of Health Guide for the Care and Use of Laboratory Animals (publication no. 85-23, revised 1996) and approved by the Institutional Animal Care and Use Committee of Nanjing Medical University (NJMU/IACUC-0402001). An unbiased approach (using randomization and blinded analyses, comprehensive phenotypical characterization of animal models, standardization of experimental conditions, confirmation of in vivo findings in $>1$ animal model; use of meaningful end points, and so on) described by Humbert et al. (16) group was performed in the present in vivo study.

\section{Pulmonary arterial and $R V$ remodeling induced by MCT}

After 1-week stay in the lab environment, 8 of 40 SD rats were randomly assigned into MCT-d0 group with an intraperitoneal injection of $0.8 \mathrm{~mL}$ saline, and the rest were given an intra-peritoneal injection of MCT $(60 \mathrm{mg} / \mathrm{kg}$, Sigma-Aldrich, St. Louis, MO, USA). On day 3, 7, 14 and 28 after MCT injection, 6 to 8 rats were randomly picked out for the following examination.

\section{$R V$ remodeling induced by pulmonary artery banding $(P A B)$}

Thoracotomy was operated according to the protocol described by Bogaard et al. (17). Briefly, in male SD rats, anesthesia is induced by $5 \%$ isoflurane inhalation and maintained by $2 \%$ isoflurane inhalation in oxygen-enriched room air. Then the rats in a supine position were intubated, followed by mechanical ventilation with a volumecontrolled respirator. Positive end-expiratory pressure was maintained at $4 \mathrm{cmH}_{2} \mathrm{O}$. A left thoracotomy was performed, and the pulmonary artery trunk was dissected from the aorta. A silk thread was positioned under the pulmonary artery trunk, and an 18-gauge needle was placed along the pulmonary artery trunk. A tight knot was made around the 18-gauge needle, and the needle was drawn out rapidly to provide a fixed opening in the lumen with a diameter of the 
needle. After the PAB, the thorax was sutured layer by layer, and postoperative pain was relieved with buprenorphine (15 $\mu \mathrm{g} / \mathrm{kg}$, s.c.). On day 28 , rats were harvested for the analysis of $\mathrm{RV}$ remodeling. With severe $\mathrm{PAB}$, the mortality reached $50 \%$ in adult rodents.

\section{Hemodynamic measurement and morphological analysis}

Eight rats were randomly picked up on day 0, 3, 7, 14 and 28 , thereafter, anesthetized using urethane $(1.0 \mathrm{~g} / \mathrm{kg}$, intraperitoneal) and mean pulmonary arterial pressure (mPAP) along with RV systolic pressure (RVSP) were measured by right-heart catheterization as described in a previous study (18). After rats were sacrificed, the heart was isolated from the thorax, and the RV free wall was dissected from the left ventricle (LV) and septum. Weight of the $\mathrm{RV}$, free left ventricular wall and ventricular septum was determined. Right ventricular hypertrophy index (RVHI) was calculated as the weight ratio of $\mathrm{RV} /[\mathrm{LV}+$ interventricular septum (IVS)]. After the calculation of RVHI, the RV and the left lung were immediately fixed in $4 \%$ paraformaldehyde $(\mathrm{pH} 7.4)$ for $24 \mathrm{~h}$, and embedded in paraffin, then sliced into 4-6 $\mu \mathrm{m}$ sections. The left lung and RV were processed with hematoxylin and eosin (HE) staining after deparaffinization. The average cross section area (CSA) of 200 myocardial cells from each section was calculated by image pro plus 6.0.

\section{Gomori aldehyde fuchsin (GAF) staining}

GAF staining was performed according to manufacturer's instructions. Both elastic fibers and MCs were selectively stained dark purple. A total number of 15 prealveolar pulmonary arteries (outer diameter of $50-150 \mu \mathrm{m}$ ) in each section were assessed at 400 magnification. Pulmonary arterial wall thickness (PAWT) was calculated according to the following formula: (external diameter - internal diameter)/external diameter (19).

\section{MCs histochemical staining}

The density and degranulation of MCs were analyzed by toluidine blue (TB) staining. After deparaffinization, the sections were soaked in a solution of $1 \%$ TB for $2-3 \mathrm{~min}$, pH 2.2 (Sigma, Munich, Germany). Then sections were dehydrated in $96-100 \%$ ethanol for 20-30 s, dipped in xylene, and finally cover-slipped. MCs density was quantified by counting the number of TB-positive MCs with a Leica 2500 microscope (Leica Microsystems, Wetzlar, Germany) at 400 magnification. In addition, degranulated MCs (light cytoplasm with empty spots) were separated from granulated MCs (intact MCs with dense cytoplasm) based on the extrusion of secretory granules (13). Moreover, an index of granulation (IOG) [(number of granulated MCs/number of degranulated MCs)] was determined.

\section{Picrosirius red (PSR) staining}

Sections were covered with saturated aqueous picric acid for $1 \mathrm{~min}$, then stained in $0.4 \%$ PSR solution $(0.4 \mathrm{~g}$ sirius red $\mathrm{F} 3 \mathrm{~B}$ combined with $100 \mathrm{~mL}$ saturated aqueous picric acid) for $1 \mathrm{~min}$. After that, sections were dehydrated in $100 \%$ ethanol, and dipped in xylene, finally cover-slipped. For each ventricle, a minimum of 12 randomly chosen areas were analyzed by microscopy at 400 magnification. Amount of red (collagen) and yellow (tissue) pixels were analyzed with Image-Pro Plus 6.0 to determine the content of fibrosis for each of the areas [red pixels/(red + yellow pixels)] (20).

\section{Immunobistochemistry}

Immunohistochemical staining was performed according to the common protocol $(21,22)$ with primary antibodies against alpha smooth muscle actin ( $\alpha$-SMA), S100A4, tryptase and CD68 (Abcam, Cambridge, MA). Through positive immunoperoxidase staining with an antibody against $\alpha$-SMA, the numbers of partially and fully muscularized distal pulmonary vessels from every ten $\times 200$ magnification fields were calculated. Moreover, tryptase was used to further recognize MCs when CD68 positive cells were considered as activated tissue macrophages.

\section{Statistical analysis}

Values were presented as the mean \pm standard error of the mean (SEM). Differences between two groups were analyzed by a two-tailed Student's $t$-test. Wherever appropriate, oneway ANOVA followed by Dunnett T3 post hoc test was used to determine differences between the mean values of different groups. It was considered statistically significant when $\mathrm{P}<0.05$.

\section{Results}

\section{Temporal alterations of morphology in pulmonary circulation after MCT injection}

After MCT challenge, evaluated mPAP (Figure 1A) as well 

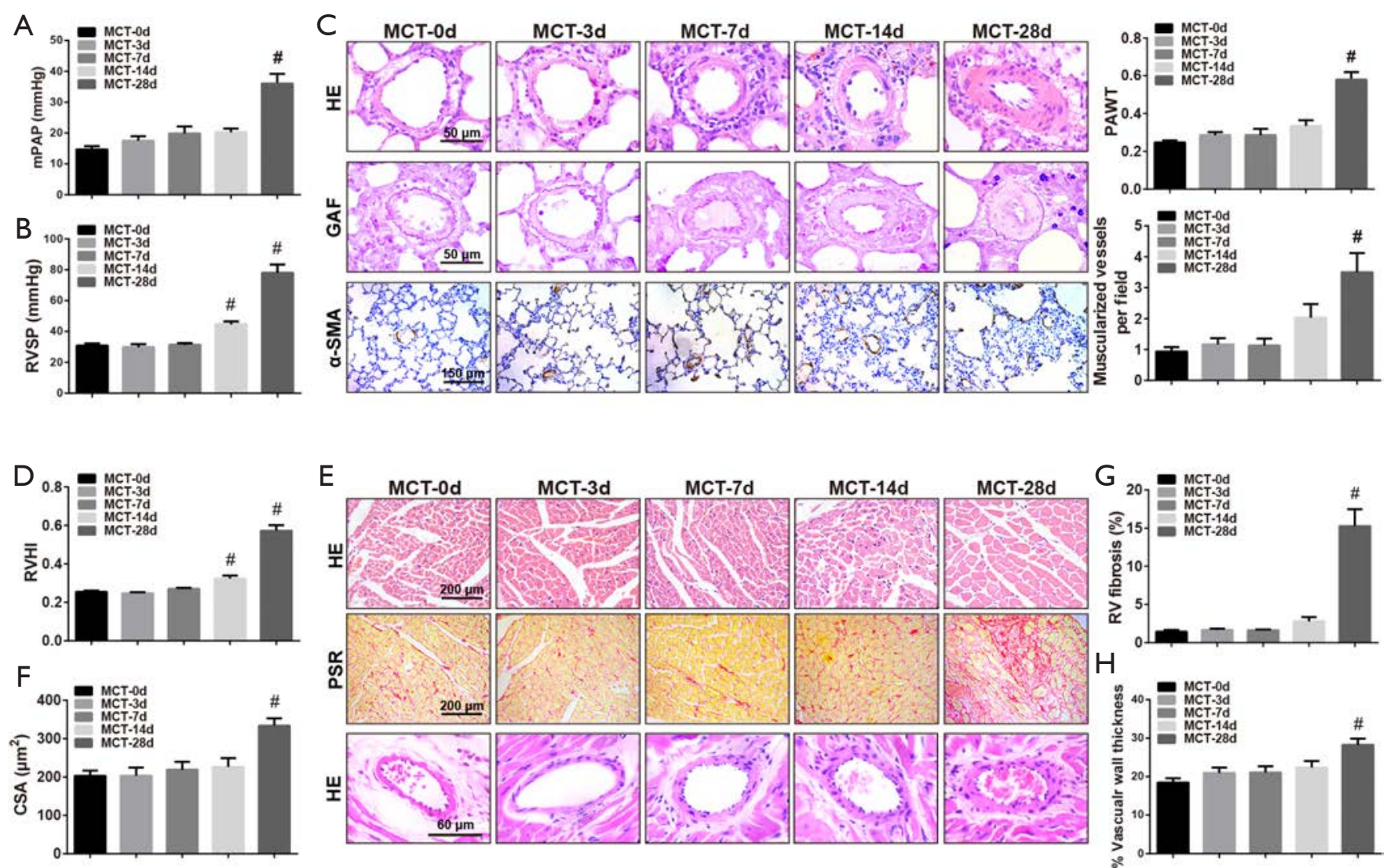

Figure 1 Temporal alternations of morphology in pulmonary circulation after monocrotaline (MCT) injection. Rat lung and right ventricular (RV) tissues were harvested on day $0,3,7,14$ and 28 after MCT challenge (60 mg/kg) for hemodynamic measurement and morphological analysis. (A) Mean pulmonary arterial pressure (mPAP); (B) right ventricular systolic pressure (RVSP); (C) representative photomicrographs from lung tissues with HE staining, Gomori aldehyde fuchsin (GAF) staining and $\alpha$-SMA staining (left panel), pulmonary artery wall thickness (PAWT) was calculated according to GAF, and muscularized vessels were counted by $\alpha$-SMA staining; (D) RV hypertrophy index (RVHI); (E) representative photomicrographs from RV tissues with HE staining and picrosirius red (PSR) staining; (F) cross section area (CSA) was calculated via HE staining; (G) RV fibrosis was evaluated using PSR staining; (H) hypertrophy of coronary artery medial layer. Data are presented as the mean \pm SEM $(n=6-8)$. ${ }^{*}, \mathrm{P}<0.05$ compared to MCT-0d. SEM, standard error of the mean.

as RVSP (Figure 1B) was observed in rats. RVSP increased significantly on day 14 and 28 when mPAP on day 28. According to HE staining, hypertrophy of pulmonary arterial media developed with time after MCT injection (Figure 1C). PAWT, the index of medial hypertrophy of muscular arteries determined by GAF staining, increased significantly at the time point of day 28 after MCT injection. Corresponding to the tunica medial hypertrophy occurred in muscular arteries, the muscularization level of precapillary arteries increased gradually. The density of muscularized distal pulmonary vessels increased significantly on day 28 .

Besides, the index concerning RV hypertrophy (RVHI) (Figure 1D) showed a significant increase on day 14 and
28, which means the remodeling of RV. Furthermore, HE staining and PSR staining were employed to evaluate the alterations in RV structure of MCT-treated rats (Figure 1E). As expected, myocardial hypertrophy (CSA), RV fibrosis, and coronary wall hypertrophy were demonstrated statistical significance on day 28 after MCT injection (Figure $1 F, G, H$ ).

\section{Spatiotemporal distribution, density and degranulation of MCs in lung tissues of MCT-treated rats}

According to the TB staining in rat lung tissues from MCTOd group, several MCs were scattered in the subpleural, adventitia of large vessels and peribronchial area, but rarely in 
A

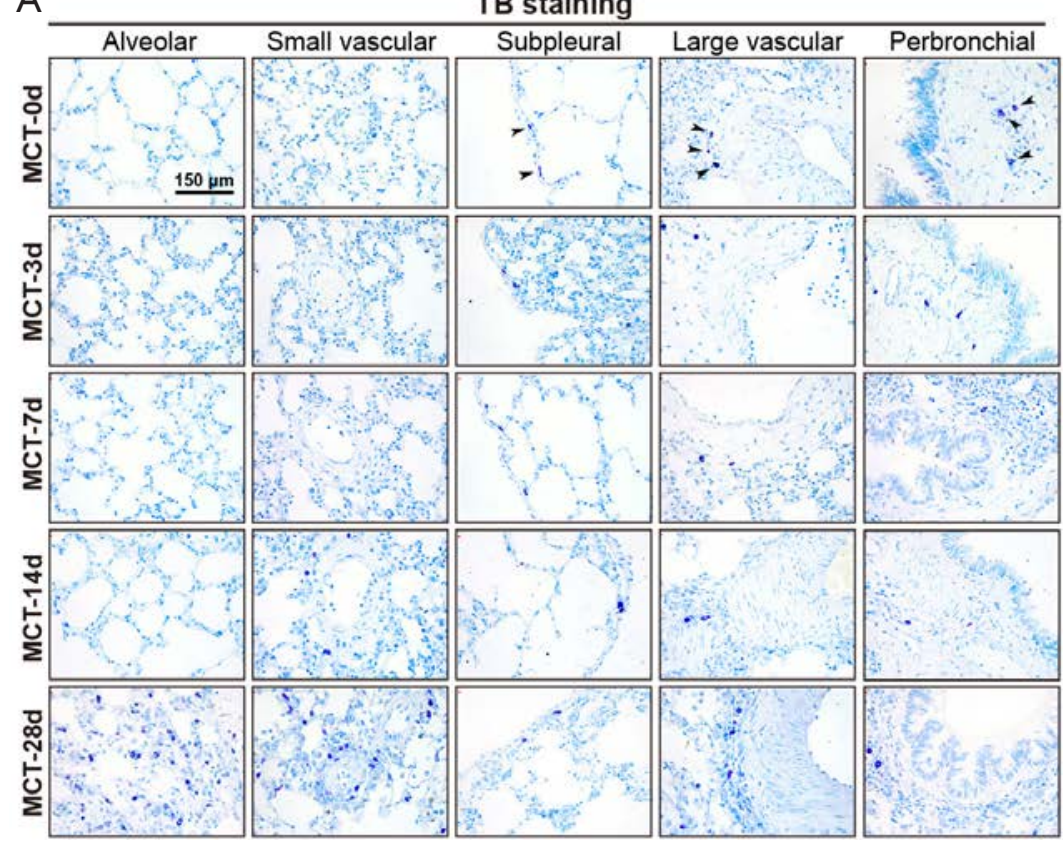

B

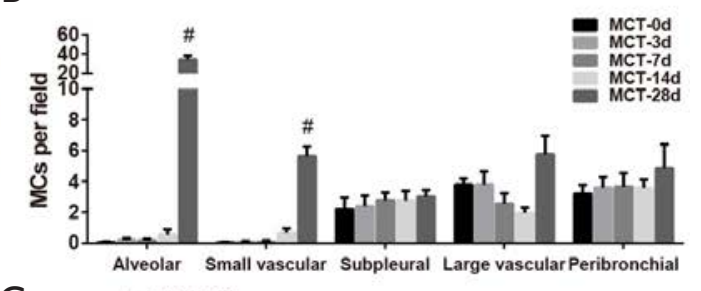

C
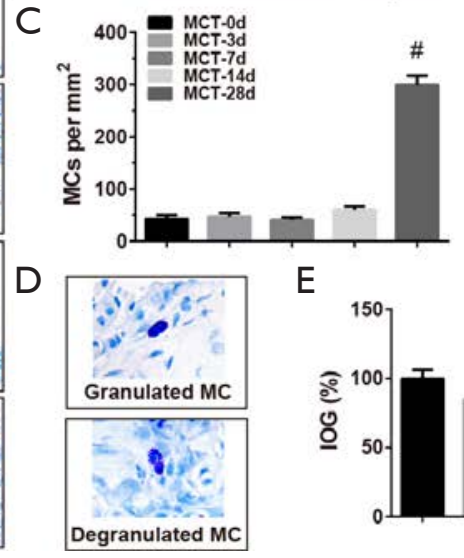

$\mathrm{E}$

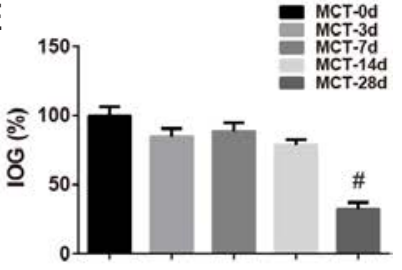

Figure 2 Spatiotemporal distribution, density and degranulation of mast cells (MCs) in lung tissues from MCT-treated rats. (A) Lung tissues were harvested for morphological analysis by toluidine blue (TB) staining, the arrow indicates the positive cells (MCs); (B,C) number of MCs in each field was calculated at 400 magnification; (D) representative photomicrographs of granulated MC and degranulated MC; (E) index of granulation (IOG) of MCs. Data are presented as the mean \pm SEM ( $n=6-8)$. ", $\mathrm{P}<0.05$ compared to MCT-0d. SEM, standard error of the mean.

alveolar septa or small muscular vessels (Figure $2 A, B$ ). On day 3 and 7 after MCT injection, the distribution and the density of MCs differed from those of MCT-0d without significance. On day 14 after MCT injection, a few MCs infiltrated into the adventitia of small muscular vessels, not remarkably. But on day 28, a number of MCs accumulated around intraacinar arteries, and infiltrated into alveolar septa. However, no significant changes in density of MCs in subpleural, adventitia of large vessels or peribronchial location were observed. The density of MCs in lungs increased significantly on day 28 after MCT injection (Figure 2C). TB staining was also used to separate degranulated MCs from granulated MCs (Figure 2D). The indexes about granulated MCs differed from those in normal rats remarkably on day 28 , but not on day 3, 7 and 14 (Figure 2E).

\section{Spatiotemporal distribution and density of MCs in lung tissues were similar to CD68-positive macrophages}

As key inflammatory cells in PAH, activated macrophages, labeled by $\mathrm{CD} 68$, facilitate pulmonary vascular remodeling. In $\mathrm{PAH}$, tryptase produced by MCs induced abnormal proliferation of vascular cells, including vascular smooth muscle cells (VSMCs) and fibroblasts. In the present study, MCs were also labeled by primary antibody against tryptase when macrophages were labeled by primary antibody against CD68 (Figure 3A). The results of MCs with tryptase staining agreed with theses of TB staining (Figure 3B). Meanwhile, most CD68+ macrophages were located in subpleural and adventitia of large vessels and peribronchial (Figure 3C) lung tissues of rats from MCT-d0 group, and some few in alveolar septa and distal muscular vessels. CD68-positive macrophages accumulated into adventitia of distal arteries gradually on day 3 and 7. The density of CD68+ macrophages increased remarkably on day 14 and 28 in small muscular vessels and alveolar septa. These changes in CD68-positive macrophages were similar to those observed in tryptase-positive MCs.

\section{Spatiotemporal distribution and density of MCs in $R V$ tissues of MCT-treated rats}

According to TB staining in rats' RV from MCT-d0 group, MCs were sparsely distributed in epicardium, myocardial interstitium and perivascular area (Figure $4 A$ ). On day 3 , 
A

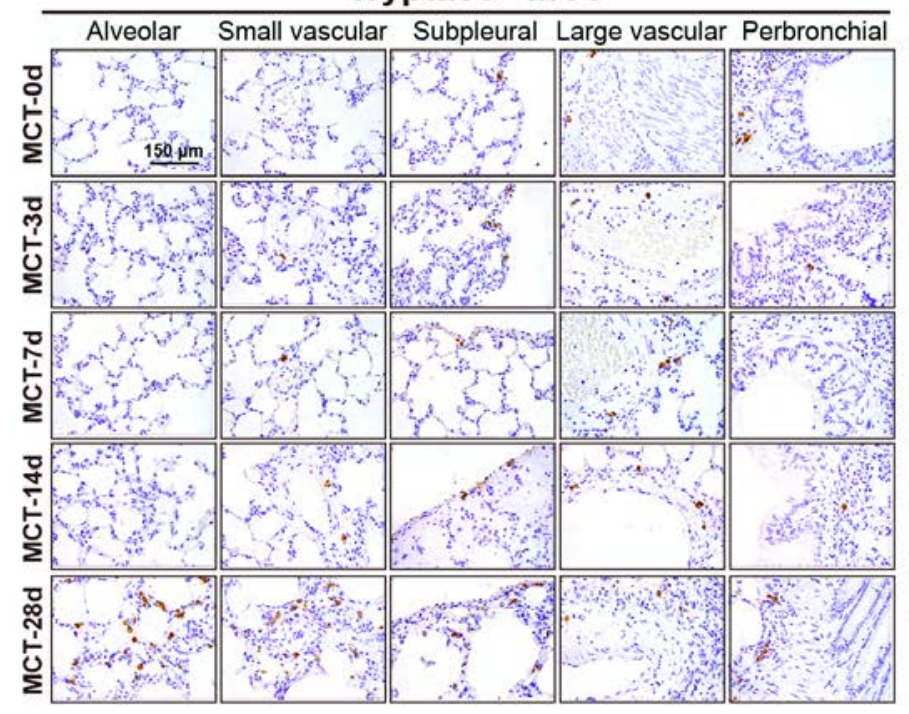

B

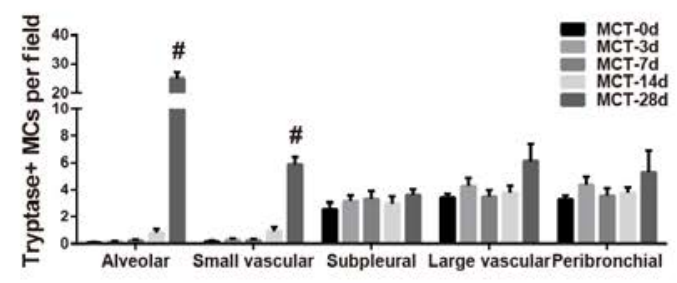

CD68+ macrophages

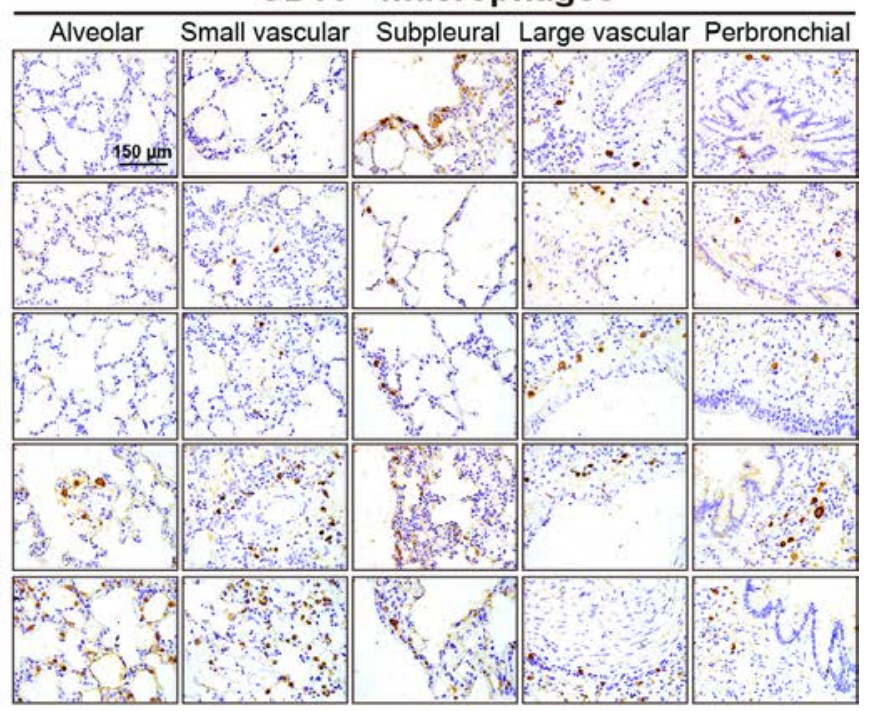

C $\frac{0}{9}$

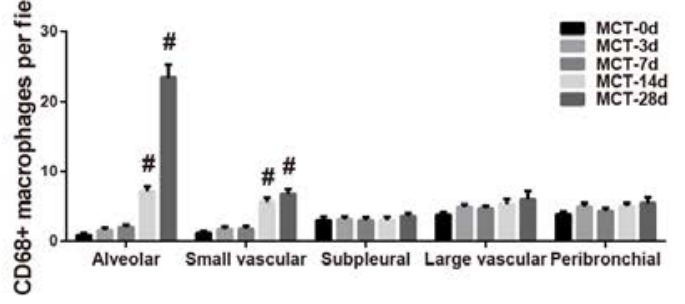

Figure 3 Spatiotemporal distribution and density of MCs in lung tissues were similar to CD68 positive macrophages. (A) Immunohistochemistry for MCs reacting to tryptase and macrophages reacting to CD68; (B) graphic analysis of tryptase staining; (C) graphic analysis of CD68 staining. Data are presented as the mean \pm SEM ( $\mathrm{n}=6-8)$. *, $\mathrm{P}<0.05$ compared to MCT-0d. MC, mast cell; SEM, standard error of the mean.

7 and 14 after MCT injection, the distribution and the density of MCs had no significant difference from those in control group. On day 28, no increase of MCs was found in the inflammatory infiltration area and the fibrosis area. The graphic analysis indicated no remarkable changes in the distribution and density of MCs in MCT-treated rats' RV. To further confirm the results above, immunoperoxidase staining with an antibody against tryptase (Figure $4 B$ ) and CD68 (Figure 4C) was performed. The data showed that neither tryptase+ MCs nor CD68+ macrophages redistributed or accumulated in RV tissues after MCT injection in rats.

\section{Spatiotemporal distribution, density and degranulation of MCs in RV tissues in PAB-operated rats}

To further investigate the association between MCs and RV remodeling, we simulated the mechanical stress brought by elevated pulmonary artery pressure with $\mathrm{PAB}$ to induce $\mathrm{RV}$ remodeling. As is presented in Figure 5A, PAB induced more obvious RV hypertrophy, myocardial hypertrophy (Figure 5B) and fibrosis (Figure 5C) when compared to those in sham operation group (Sham). In RV tissues of sham rats, MCs were sparsely distributed in epicardium, myocardial interstitium and perivascular area. However, neither the distribution nor the density of MCs in RV tissues of PAB-treated rats differed significantly from those in sham group according to the GAF staining (Figure $5 D$ ). The result of MCs degranulation (IOG) made no difference between $\mathrm{PAB}$ rats and sham rats (Figure 5E). Moreover, the distribution and density of tryptase-positive MCs (Figure $5 F$ ) were identical to those of CD68-positive macrophages (Figure 5G) in RV tissues, showing no remarkable difference in RV tissues between $\mathrm{PAB}$-treated rats and sham group rats. 

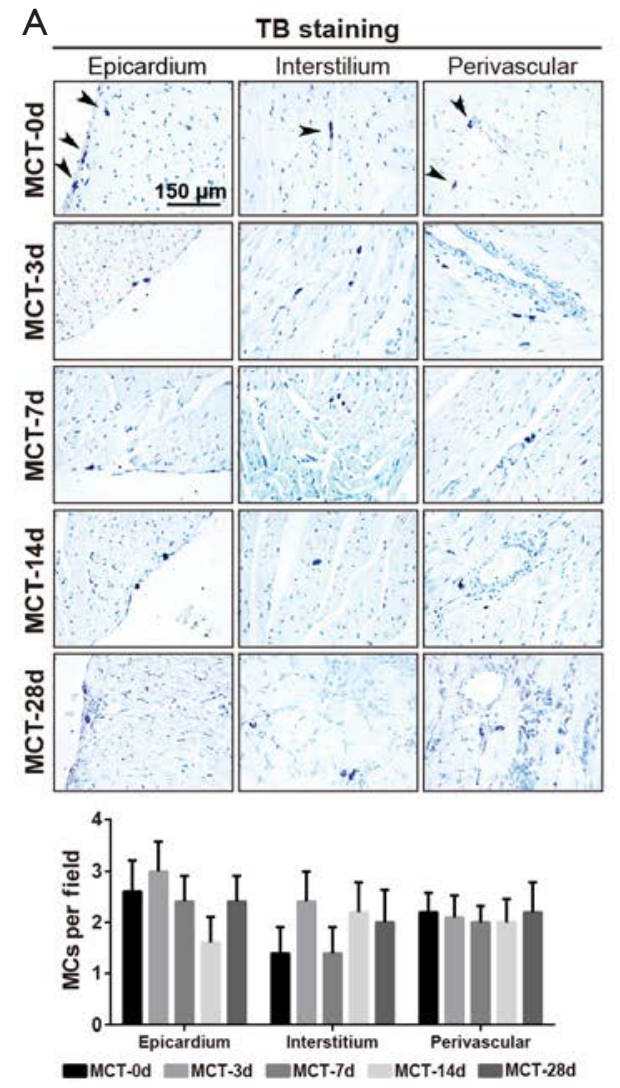

B
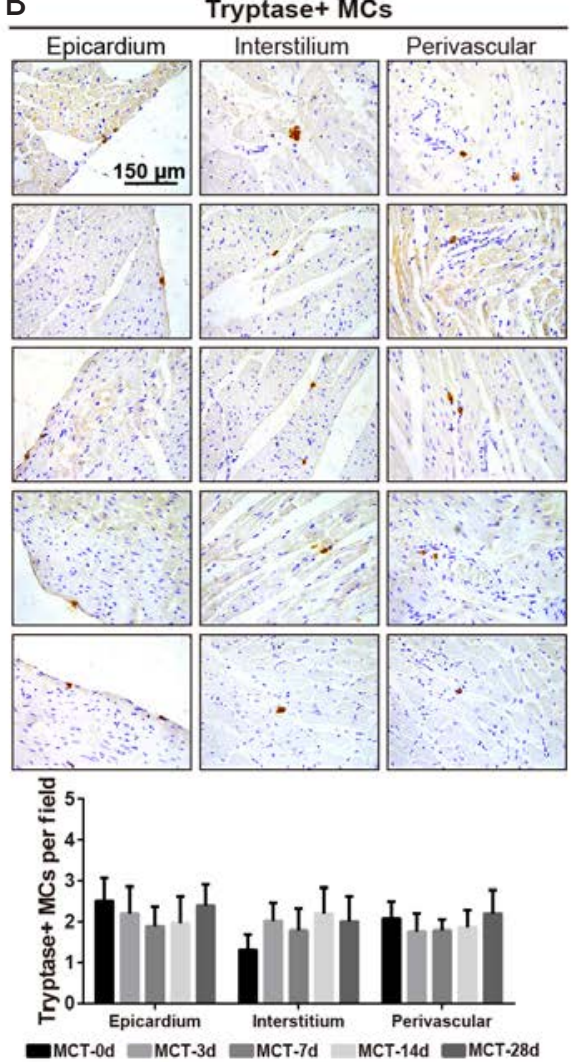
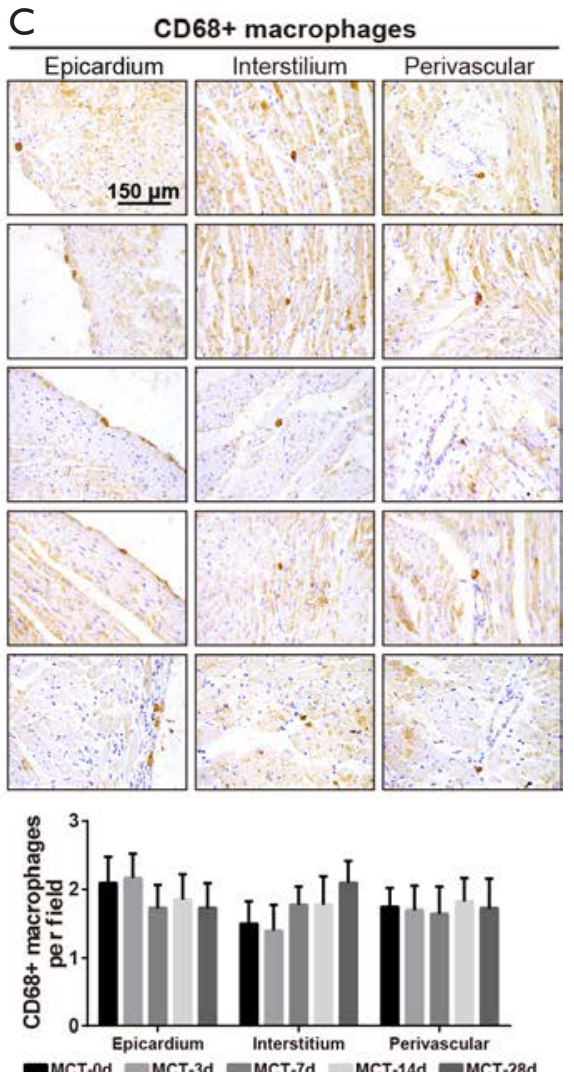

Figure 4 Spatiotemporal distribution and density of MCs and macrophages in RV. (A) TB staining; (B) immunohistochemical staining with primary antibody against tryptase; (C) immunohistochemical staining with primary antibody against tryptase. Data are presented as the mean \pm SEM ( $n=6-8)$. MC, mast cell; RV, right ventricle; TB, toluidine blue; SEM, standard error of the mean.

\section{Discussion}

MCs are extensively observed around pulmonary vessels in $\mathrm{PAH}$. However, their temporal and spatial variation during the development of PAH remains obscure. Little is known about the role of MCs in RV remodeling. On the basis of the present study, the crucial role of MCs in pulmonary vascular remodeling was revealed in detail, laying a conclusion that MCs had no relevance to RV remodeling.

MCT model and chronic hypoxia model (CHP) were the commonly employed experimental PAH animal models (23). In MCT-PAH, the endothelial injury is the initial trigger for pulmonary vasculitis and obstructive pulmonary vascular remodeling, characterized by narrowing/obliteration of the vascular lumen. It could also highlight the pivotal role of inflammatory cells (macrophages, dendritic cells, and MCs). However, The CHP model is assumed to be representative of group 3 ( $\mathrm{PH}$ secondary to lung disease and/or hypoxia) $(3,24)$. CHP cannot fully recapitulate the pulmonary vascular damage (including infiltration of inflammatory cells) observed in humans with $\mathrm{PH}$. CHP combined with SU-5416 (an inhibitor of the vascular endothelial growth factor receptor-2) (25) can cause $\mathrm{PH}$ with pulmonary arterial changes resembling plexiform lesions, and is mainly used to study the angioproliferative features of PH $(26,27)$. Taken together, MCT model was used in the present study to explore the role of MCs in PAH.

In lungs from control rats, a small number of MCs were scattered in the subpleural and adventitia of large vessels and peribronchial area, but rarely in alveolar septa or small muscular vessels. This founding was consistent with the results stated by Vajner et al. (8). The infiltration of MCs into intra-acinar arteries and alveolar septa continued on day 3, 7 and 14, and the remarkable accumulation of MCs only occurred on day 28 after MCT injection. Meanwhile, MCs in lung tissues saw the same turning points of degranulation. So, the typical characterizations 
A
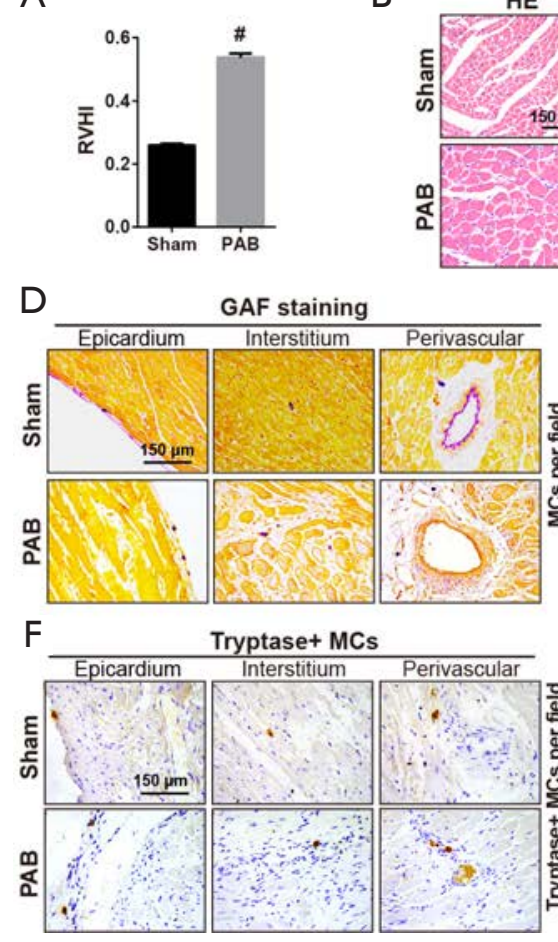

B

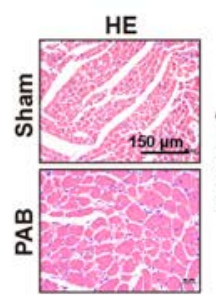

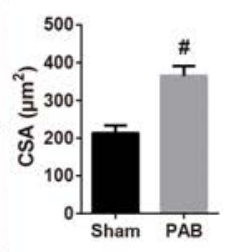

C

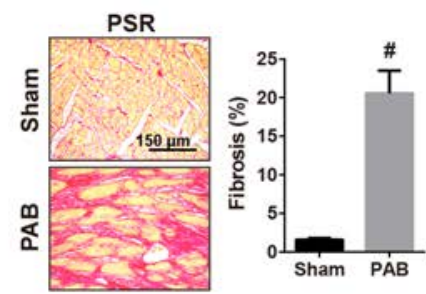

$\mathrm{E}$
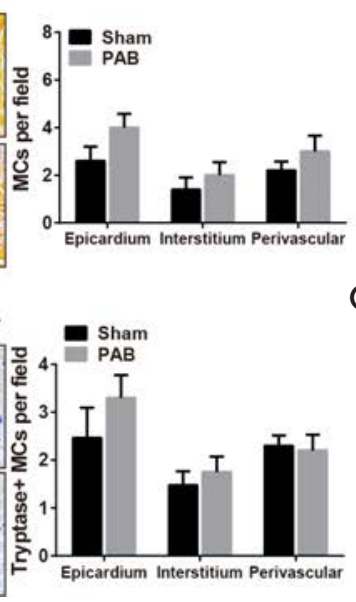
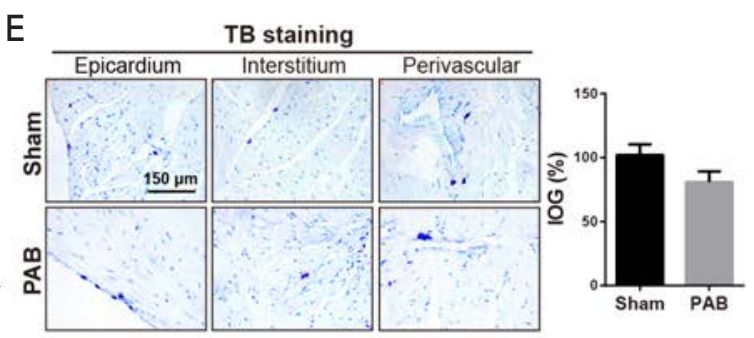

G CD68+ macrophages

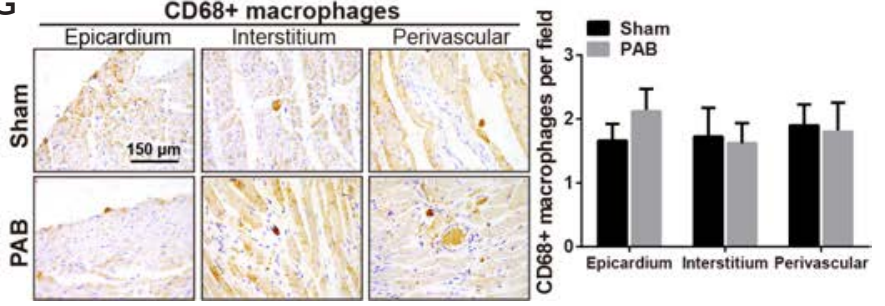

Figure 5 Distribution, density and degranulation of MCs in RV tissues in pulmonary artery banding (PAB) rats. (A) RVHI; (B) representative photomicrographs from RV tissues with HE staining tissues and CSA; (C) PSR staining and RV fibrosis; (D) GAF staining; (E) TB staining and IOG; (F) immunohistochemical staining with primary antibody against tryptase; (G) immunohistochemical staining with primary antibody against CD68. Data are presented as the mean $\pm \mathrm{SEM}(\mathrm{n}=6-8)$. ", $\mathrm{P}<0.05$ compared to sham. MC, mast cell; RV, right ventricle; TB, toluidine blue; RVHI, right ventricular hypertrophy index; CSA, cross section area; PSR, picrosirius red; IOG, index of granulation; SEM, standard error of the mean.

on day 28 were the infiltration and degranulation of MCs in alveolar septa and small arteries. To confirm our data about MCs' infiltration, we compared our study with those reported previously. Our findings were consistent with those stated by Vaszar et al. (28). In the rat model of pneumonectomy-MCT induced PAH, the expression of MCs-related mRNA increased gradually and remarkably from day 28 after MCT injection. Among these, mast cell protease- 1 increased by 21 times in mRNA expression, mast cell protease- 7 by 4.7 times, and mast cell chymase- 1 by 10 times. These results were also similar to those occurred in rats exposed to hypoxia (8). The total number of MCs in lung tissues showed an increase in rats exposed to hypoxia when the lung vascular remodeling occurred obviously, but no significant increase was observed before the vascular remodeling occurred. So, the infiltration of MCs around pulmonary vessels was accompanied by pulmonary vascular remodeling.
Accompanying with pulmonary vascular remodeling, similar pathological changes happened in RV including hypertrophy, inflammation and fibrosis. RV remodeling and pulmonary vascular remodeling share several molecular mechanisms, including endoplasmic reticulum stress (18), oxidative stress and mitochondrial stress. According to the data available in literature, MCs contribute to cardiac hypertrophy (29) and cardiac fibrosis (30) in $\mathrm{LV}$. In response to MCT, systemic procoagulant and proinflammatory responses cause coronary arteriolar thickening, myocarditis and eventual RV remodeling with increased afterload (31). Taken together, MCs seem to be involved in RV remodeling apart from pulmonary vascular remodeling. Unexpectedly, the present study found no changes in the distribution, density and degranulation of MCs in RV after MCT injection. Besides, hypertrophy of coronary artery medial layer was noted in MCTtreated rats on day 28. It was consistent with the results 
described by Meloche et al (32). In the present article, coronary artery location was described as perivascular area. Nevertheless, infiltration of MC into perivascular area/ coronary artery, was not observed. Which indicates that MC might not be involved in coronary artery remodeling in MCT-PAH rats. Moreover, we imposed the simulated pressure with $\mathrm{PAB}$ to induce $\mathrm{RV}$ remodeling, but observed no remarkable difference in the distribution, density and degranulation of MCs in RV. These data proved that MCs did not contribute to RV remodeling. Whether MCs in lung tissues affect $\mathrm{RV}$ remodeling via telecrine needs further exploration.

Since CD68-positive macrophages are key inflammatory cells contributing to pulmonary vascular remodeling, we compared the distribution and density of MCs with those of CD68-positive macrophages in lung tissues. In the present study, the density of CD68-positive macrophages increased slightly on day 3 and 7 in lung tissues, and increased significantly on day 14 and 28. Most of them moved into alveoli and perivascular regions. This was consistent with the finding of Kimura et al. (33). Combining the distribution and density of MCs with those of macrophages, MCs and macrophages might play the same role in vascular remodeling. And the accumulation of MCs in lung tissues might be regulated by macrophages due to the result that macrophages accumulated prior to MCs in lung tissues and the fact that MCs can be recruited and activated by monocyte chemoattractant protein-1 derived from macrophages (34). This assumption needs to be confirmed with more experiments.

According to Rabinovitch's morphometric classification of pulmonary vascular remodeling in PAH (35), morphometric grade A is about an abnormality with the extension of muscles into peripheral and normally nonmuscular arteries, morphometric grade B about a structural remodeling with the thickness of medial wall increased by $\geq 1.5$ times, and morphometric grade $\mathrm{C}$ about the reduction of arterial concentration and artery size. In the present study, the remodeling of pulmonary vasculatures on day 3, 7 and 14 belonged to morphometric grade A and that on day 28 belonged to morphometric grade B. Therefore, the infiltration of MCs in lung tissues occurred in the period of morphometric grade B. The accumulation of MCs was related to the hypertrophy of vascular media in morphometric grade B rather than in morphometric grade A. These data agreed with the results obtained by Stacher et al. (36) that perivascular inflammation was correlated with fractional thickness of media and adventitia. Thus, we concluded that MCs contributed to the remodeling in morphometric grade $\mathrm{B}$ rather than that in morphometric grade A. Whether MCs contributed to the reduction of arterial concentration in morphometric grade $\mathrm{C}$ needs to be proved by further studies.

The cytokines that mediate the effect of MCs on pulmonary vascular remodeling might be MCs-derived tryptase (15) or others. On the basis of the present study, further experiment should be performed to explore the effect of different MCs-derived cytokines on pulmonary vascular remodeling for an extended understanding of the underlying mechanisms of PAH.

MCT model is a commonly employed experimental $\mathrm{PAH}$ animal model (23). And the histological investigation of the pulmonary vasculature in $\mathrm{PAH}$ shows features such as intimal hyperplasia, medial hypertrophy, and adventitial inflammatory infiltration $(3,24)$. In addition to intimal hyperplasia and medial hypertrophy, plexiform lesions, angiomatoid lesions and fibrinoid necrosis are also the main histological features of group $1 \mathrm{PAH}$ in human beings. Nevertheless, these histological features are rarely observed in MCT model. Therefore, MCT model does not reflect entirely the pathophysiological process of group $1 \mathrm{PAH}$ in humans (37). Many efforts need to be made to explore the role of MCs in different experiment $\mathrm{PAH}$ model. Besides, the present study provided an association between MC and the development of pulmonary arterial wall hypertrophy. However, the causal relationship has not be demonstrated by the present study. Further exploration, using MC stabiliser ketotifen or MC-deficient Ws/Ws animals (10), will be performed to demonstrated the causal relationship.

The present study implied a crucial effect of MCs on the development of pulmonary vascular remodeling but not RV remodeling in PAH. MCs which were probably recruited by CD68-positive macrophages, triggered evident pulmonary vascular remodeling which probably through the MCs derived cytokines such as tryptase.

\section{Acknowledgements}

Funding: This study was supported by the National Natural Science Foundation of China (No. 81273571), the Jiangsu Clinical Research Center for Respiratory Diseases Project under Grant BL2012012, the Jiangsu Province Scientific Research Innovation Project of University graduate students (No. JX22013361), and by a project funded by the Priority Academic Program Development of Jiangsu Higher 
Education Institutions (PAPD) (JX10231802).

\section{Footnote}

Conflicts of Interest: The authors have no conflicts of interest to declare.

Ethical Statement: All procedures were performed according to the National Institutes of Health Guide for the Care and Use of Laboratory Animals (publication no. 85-23, revised 1996) and approved by the Institutional Animal Care and Use Committee of Nanjing Medical University (NJMU/ IACUC-0402001).

\section{References}

1. Humbert M, Sitbon O, Chaouat A, et al. Survival in patients with idiopathic, familial, and anorexigenassociated pulmonary arterial hypertension in the modern management era. Circulation 2010;122:156-63.

2. Vonk-Noordegraaf A, Haddad F, Chin KM, et al. Right heart adaptation to pulmonary arterial hypertension: physiology and pathobiology. J Am Coll Cardiol 2013;62:D22-33.

3. Stenmark KR, Meyrick B, Galie N, et al. Animal models of pulmonary arterial hypertension: the hope for etiological discovery and pharmacological cure. Am J Physiol Lung Cell Mol Physiol 2009;297:L1013-32.

4. Tuder R M, Stacher E, Robinson J, et al. Pathology of pulmonary hypertension. Clin Chest Med 2013;34:639-50.

5. Haas F, Bergofsky EH. Role of the mast cell in the pulmonary pressor response to hypoxia. J Clin Invest 1972;51:3154-62.

6. Tucker A, McMurtry IF, Alexander AF, et al. Lung mast cell density and distribution in chronically hypoxic animals. J Appl Physiol Respir Environ Exerc Physiol 1977;42:174-8.

7. Kay JM, Waymire JC, Grover RF. Lung mast cell hyperplasia and pulmonary histamine-forming capacity in hypoxic rats. Am J Physiol 1974;226:178-84.

8. Vajner L, Vytasek R, Lachmanova V, et al. Acute and chronic hypoxia as well as 7 -day recovery from chronic hypoxia affects the distribution of pulmonary mast cells and their MMP-13 expression in rats. Int J Exp Pathol 2006;87:383-91.

9. Kosanovic D, Dahal BK, Peters D M, et al. Histological characterization of mast cell chymase in patients with pulmonary hypertension and chronic obstructive pulmonary disease. Pulm Circ 2014;4:128-36.

10. Hoffmann J, Yin J, Kukucka M, et al. Mast cells promote lung vascular remodelling in pulmonary hypertension. Eur Respir J 2011;37:1400-10.

11. Banasova A, Maxova H, Hampl V, et al. Prevention of mast cell degranulation by disodium cromoglycate attenuates the development of hypoxic pulmonary hypertension in rats exposed to chronic hypoxia. Respiration 2008;76:102-7.

12. Bartelds B, van Loon RL, Mohaupt S, et al. Mast cell inhibition improves pulmonary vascular remodeling in pulmonary hypertension. Chest 2012;141:651-60.

13. Dahal BK, Kosanovic D, Kaulen C, et al. Involvement of mast cells in monocrotaline-induced pulmonary hypertension in rats. Respir Res 2011;12:60.

14. Wang T, Han SX, Zhang SF, et al. Role of chymase in cigarette smoke-induced pulmonary artery remodeling and pulmonary hypertension in hamsters. Respir Res 2010;11:36.

15. Kwapiszewska G, Markart P, Dahal BK, et al. PAR-2 inhibition reverses experimental pulmonary hypertension. Circ Res 2012;110:1179-91.

16. Bonnet S, Provencher S, Guignabert C, et al. Translating Research into Improved Patient Care in Pulmonary Arterial Hypertension. Am J Respir Crit Care Med 2017;195:583-95.

17. Bogaard HJ, Natarajan R, Henderson SC, et al. Chronic pulmonary artery pressure elevation is insufficient to explain right heart failure. Circulation 2009;120:1951-60.

18. Wang JJ, Zuo XR, Xu J, et al. Evaluation and Treatment of Endoplasmic Reticulum (ER) Stress in Right Ventricular Dysfunction during Monocrotaline-Induced Rat Pulmonary Arterial Hypertension. Cardiovasc Drugs Ther 2016;30:587-98.

19. Nawa N, Ishida H, Katsuragi S, et al. Constitutively active form of natriuretic peptide receptor 2 ameliorates experimental pulmonary arterial hypertension. Mol Ther Methods Clin Dev 2016;3:16044.

20. Stein M, Noorman M, van Veen TA, et al. Dominant arrhythmia vulnerability of the right ventricle in senescent mice. Heart Rhythm 2008;5:438-48.

21. Xu J, Wang J, Cheng Y, et al. Glucagon-Like Peptide-1 Mediates the Protective Effect of the Dipeptidyl Peptidase IV Inhibitor on Renal Fibrosis via Reducing the Phenotypic Conversion of Renal Microvascular Cells in Monocrotaline-Treated Rats. Biomed Res Int 2018;2018:1864107.

22. Wang J, Xu J, Zhao X, et al. Fasudil inhibits neutrophil- 
endothelial cell interactions by regulating the expressions of GRP78 and BMPR2. Exp Cell Res 2018;365:97-105.

23. Maarman G, Lecour S, Butrous G, et al. A comprehensive review: the evolution of animal models in pulmonary hypertension research; are we there yet? Pulm Circ 2013;3:739-56.

24. Ryan J, Bloch K, Archer SL. Rodent models of pulmonary hypertension: harmonisation with the world health organisation's categorisation of human PH. Int J Clin Pract Suppl 2011:15-34.

25. Ciuclan L, Bonneau O, Hussey M, et al. A novel murine model of severe pulmonary arterial hypertension. Am J Respir Crit Care Med 2011;184:1171-82.

26. Casserly B, Mazer JM, Vang A, et al. C-type natriuretic peptide does not attenuate the development of pulmonary hypertension caused by hypoxia and VEGF receptor blockade. Life Sci 2011;89:460-6.

27. Taraseviciene-Stewart L, Kasahara Y, Alger L, et al. Inhibition of the VEGF receptor 2 combined with chronic hypoxia causes cell death-dependent pulmonary endothelial cell proliferation and severe pulmonary hypertension. FASEB J 2001;15:427-38.

28. Vaszar LT, Nishimura T, Storey JD, et al. Longitudinal transcriptional analysis of developing neointimal vascular occlusion and pulmonary hypertension in rats. Physiol Genomics 2004;17:150-6.

29. Singh AP, Singh M, Balakumar P. Effect of mast cell stabilizers in hyperhomocysteinemia-induced cardiac hypertrophy in rats. J Cardiovasc Pharmacol 2008;51:596-604.

30. Kanemitsu H, Takai S, Tsuneyoshi H, et al. Chronic

Cite this article as: $\mathrm{Xu} \mathrm{J}$, Wang J, Shao C, Zeng X, Sun L, Kong H, Xie W, Wang H. New dynamic viewing of mast cells in pulmonary arterial hypertension $(\mathrm{PAH})$ : contributors or outsiders to cardiovascular remodeling. J Thorac Dis 2018;10(5):3016-3026. doi: 10.21037/jtd.2018.05.59 chymase inhibition preserves cardiac function after left ventricular repair in rats. Eur J Cardiothorac Surg 2008;33:25-31.

31. Guihaire J, Bogaard HJ, Flecher E, et al. Experimental models of right heart failure: a window for translational research in pulmonary hypertension. Semin Respir Crit Care Med 2013;34:689-99.

32. Meloche J, Lampron MC, Nadeau V, et al. Implication of Inflammation and Epigenetic Readers in Coronary Artery Remodeling in Patients With Pulmonary Arterial Hypertension. Arterioscler Thromb Vasc Biol 2017;37:1513-23.

33. Kimura H, Kasahara Y, Kurosu K, et al. Alleviation of monocrotaline-induced pulmonary hypertension by antibodies to monocyte chemotactic and activating factor/monocyte chemoattractant protein-1. Lab Invest 1998;78:571-81.

34. Alam R, Kumar D, Anderson-Walters D, et al. Macrophage inflammatory protein-1 alpha and monocyte chemoattractant peptide-1 elicit immediate and late cutaneous reactions and activate murine mast cells in vivo. J Immunol 1994;152:1298-303.

35. Rabinovitch M. Pathobiology of pulmonary hypertension. Annu Rev Pathol 2007;2:369-99.

36. Stacher E, Graham BB, Hunt JM, et al. Modern age pathology of pulmonary arterial hypertension. Am J Respir Crit Care Med 2012;186:261-72.

37. Gomez-Arroyo JG, Farkas L, Alhussaini AA, et al. The monocrotaline model of pulmonary hypertension in perspective. Am J Physiol Lung Cell Mol Physiol 2012;302:L363-9. 